\title{
MicroRNA-26a protects against cardiac hypertrophy via inhibiting GATA4 in rat model and cultured cardiomyocytes
}

\author{
YAN LIU, ZHIQIAN WANG and WENLIANG XIAO
}

Department of Cardiology, The Third Hospital of Hebei Medical University, Shijiazhuang, Hebei 050051, P.R. China

Received September 11, 2015; Accepted July 12, 2016

DOI: $10.3892 / \mathrm{mmr} .2016 .5574$

\begin{abstract}
Pathological cardiac hypertrophy is characterized by deleterious changes developed in cardiovascular diseases, whereas microRNAs (miRNAs) are involved in the mediation of cardiac hypertrophy. To investigate the role of microRNA-26a (miR-26a) in regulating cardiac hypertrophy and its functioning mechanisms, overexpression and suppression of miR-26a via its mimic and inhibitor in a transverse abdominal aortic constriction (TAAC)-induced rat model and in angiotensin II (Ang II)-induced cardiomyocytes (CMs) was performed. In the rat model, the heart weight ( $\mathrm{HW}$ ) compared with the body weight (BW), the CM area, and expression of the hypertrophy-associated factors, atrial natriuretic factor (ANF) and $\beta$-myosin heavy chain $(\beta-\mathrm{MHC})$, were assessed. In $\mathrm{CMs}$, the protein synthesis rate was determined using a leucine incorporation assay. Mutation of the GATA-binding protein 4 (GATA4) 3'-untranslated region (UTR) and overexpression of GATA4 were performed to confirm whether GATA4 is the target of miR-26a. The results indicated that miR-26a was significantly downregulated in the heart tissue of the rat model, as well as in Ang II-induced CMs $(\mathrm{P}<0.05)$. The TAAC-induced rat model exhibited a higher HW/BW ratio, a larger $\mathrm{CM}$ area, and higher expression levels of $A N F$ and $\beta$-MHC. CMs, upon Ang II treatment, also demonstrated a larger $\mathrm{CM}$ area, higher levels of $A N F$ and $\beta-M H C$, as well as accelerated protein synthesis. miR-26a was not able to regulate GATA4 with mutations in the 3'-UTR, indicating that GATA4 was the direct target of miR-26a. Overexpression of GATA4 abrogated the inhibitory functions of miR-26a in cardiac hypertrophy. Taken together, the present study suggested an anti-hypertrophic role of miR-26a in cardiac hypertrophy, possibly via inhibition of GATA4. These findings may be useful in terms of facilitating cardiac treatment, with potential therapeutic targets and strategies.
\end{abstract}

Correspondence to: Dr Zhiqian Wang, Department of Cardiology, The Third Hospital of Hebei Medical University, 139 Ziqiang Road, Qiaoxi, Shijiazhuang, Hebei 050051, P.R. China

E-mail: wangzhiqian211_24@126.com

Key words: cardiac hypertrophy, microRNA-26a, GATA4, cardiomyocyte

\section{Introduction}

Cardiac hypertrophy occurs as a pathological process associated with a number of cardiovascular diseases, including hypertrophic cardiomyopathy (1), hypertension (2) and myocardial infarction (3). Unlike an athlete's heart, pathological cardiac hypertrophy is the response to stress or diseases, leading to increases in myocardial protein synthesis and cell volume, instead of the enhancement of pumping ability (4). Pathological cardiac hypertrophy under long-term stress may develop into cardiomegaly, heart failure, and even sudden cardiac mortality. Signaling pathways involved in the pathogenesis of cardiac hypertrophy have been investigated in recent studies. For example, inhibition of the Raf-1/mitogen-activated protein kinase kinase (MEK)/extracellular-signal-regulated kinase (ERK) cascade suppresses protein synthesis and the expression of brain natriuretic peptide in cardiac hypertrophy (5). Small GTP-binding proteins, protein kinase $\mathrm{C}$ and signal transduction and activation of transcription-3 (STAT3) are also vital regulatory factors of cardiac hypertrophy-associated genes (6-8).

The latest research has revealed significant roles of microRNAs (miRNAs) in mediating cardiac hypertrophy. As small non-coding RNAs, miRNAs regulate gene silencing post-transcriptionally, suppressing translation and inducing messenger RNA (mRNA) degradation, by binding to the 3'-untranslated region (UTR) of mRNAs (9). Overexpression of miR-208a in the heart induces cardiac hypertrophy (10), the identical regulatory pattern also being reported for miR-23a and miR-22 $(11,12)$. Several miRNAs exert anti-hypertrophic roles, such as miR-133, for example, which targets the hypertrophic regulatory factors, RhoA and $C d c 42$, to inhibit cardiac hypertrophy (13), and miR-1, whose attenuation evokes hypertrophy (14). Furthermore, miR-101 suppresses hypertrophy in the rat heart via inhibition of its target gene, Rabla (15). In general, miRNA-mediated gene expression and the regulation of further downstream signaling events exert an appreciable influence on the progression of cardiac hypertrophy, the details of which have yet to be fully elucidated.

A comprehensive study has revealed multiple miRNAs that are aberrantly expressed in cardiac hypertrophy, among which miR-26a exhibits abundant expression in the normal heart (16). In this regard, it was hypothesized that miR-26a may be an important regulator in cardiac hypertrophy, and therefore, 
in the present study, a series of experiments in a transverse abdominal aortic constriction (TAAC)-induced rat model and angiotensin II (Ang II)-induced cardiomyocytes (CMs) isolated from the neonatal rat heart were performed. miR-26a overexpression and suppression were mediated by its mimic and inhibitor, respectively. Atrial natriuretic factor (ANF) and $\beta$-myosin heavy chain ( $\beta$-MHC) were assessed as indicators of cardiac hypertrophy. Furthermore, the present study aimed to confirm whether GATA4 is the target of miR-26a via mutating the target sites in the 3'-UTR of GATA4. By means of these experiments, the present study aimed to elucidate the role of miR-26, and its regulatory mechanism, in mediating cardiac hypertrophy, which may provide leads for therapeutic targets in the treatment of cardiac hypertrophy.

\section{Materials and methods}

Animals. All the animal experiments were performed following the guidelines of our institute, and were approved by a local ethics committee. Male Sprague Dawley (SD) rats (SPF grade; Vital River Laboratories, Beijing, China) of 100-120 g were used for inducing cardiac hypertrophy using the TAAC method. The rats were maintained at $24^{\circ} \mathrm{C}$ with $50 \%$ humidity. A 12-h light/dark cycle was used and they were allowed access to sufficient standard feed and water. TAAC was performed on ten randomly chosen rat individuals (the TAAC group) and another ten individuals (the Sham group), which underwent the identical operation procedures except for TAAC. Specifically, the rats were anesthetized by intraperitoneally injecting $4 \%$ chloral hydrate solution $(60 \mathrm{mg} / \mathrm{kg})$. A vertical incision of $2-3 \mathrm{~cm}$ was made under the center abdominal xiphoid process to expose the surgical field. A ligation was made along the abdominal aorta $3 \mathrm{~mm}$ above the branch point of the right renal artery by ligating a 5-gauge needle with 4-0 sutures. Subsequently, the needle was removed and the incision was closed. Following the surgery, all the rats were treated with penicillin for 3 days. At 4 weeks post-surgery, the rats were sacrificed to examine the heart weight (HW) and body weight (BW), and also the expression levels of $A N F, \beta-M H C$ and miR-26a.

Cells. SD rats (SPF grade, Vital River Laboratories) aged 2 days old were anesthetized by inhalation of $3 \%$ diethyl ether for $1 \mathrm{~h}$ and sacrificed for heart sampling. The heart samples were immediately immersed in cold D-Hanks buffer (Sbjbio, Nanjing, China), and the ventricular muscle was isolated and minced. Subsequently, the minced tissues were incubated in trypsin (0.08\%; Sigma-Aldrich China, Inc., Shanghai, China) at $37^{\circ} \mathrm{C}$ for $5 \mathrm{~min}$. Following the termination of trypsin digestion, the cell suspension was incubated in Dulbecco's minimum essential medium (DMEM; Sigma-Aldrich China, Inc.) supplemented with $10 \%$ fetal calf serum (FCS; Sigma-Aldrich China, Inc.) and cultured in an atmosphere of $5 \% \mathrm{CO}_{2}$ at $37^{\circ} \mathrm{C}$. The medium was changed every other day. During the first $72 \mathrm{~h}$ of culture, 5-bromo-2'-deoxyuridine (0.1 mM; Sigma-Aldrich China, Inc.) was added to the medium to inhibit the growth of myocardial fibroblasts. The percentage of CMs present was determined using a mouse monoclonal anti-heavy chain cardiac myosin antibody (1:1,000; Abcam, Cambridge, UK; cat. no. ab207926) to be $>95 \%$. After $24 \mathrm{~h}$, the CMs were divided into two groups, namely the Ang II-treated group
(Ang II group) and the untreated group (Control group), and the cells were cultured in serum-free medium. Ang II $(1 \mu \mathrm{M}$; Sigma-Aldrich China, Inc.) was added to cells of the Ang II group and the cells were cultured for $48 \mathrm{~h}$, with the medium being changed every $8 \mathrm{~h}$. Subsequently, the cells were collected for further analyses.

Cell transfection and dual-luciferase reporter assay. The target gene of miR-26a was predicted using TargetScan (www.targetscan.org). The mutant 3'-UTR of GATA4 was synthesized using a QuikChange Multi Site-Directed Mutagenesis kit (Agilent Technologies, Santa Clara, CA, USA). The wild-type or mutant 3'-UTR of GATA4 was cloned into the $K p n I$ and $B g l \mathrm{II}$ restriction sites downstream of the open reading frame of Renilla luciferase in the pGL3-promoter luciferase vector (Promega, Madison, WI, USA). CMs cultured in 24-well plates $\left(1.5 \times 10^{5}\right.$ per well) were transfected with miR-26a mimic (50 nM) or inhibitor (Sangon Biotech Co., Ltd., Shanghai, China), and co-transfected with luciferase reporter containing the wild-type or mutant 3'-UTR of GATA4 (200 ng) or Renilla luciferase reporter plasmid pRL-RSV (20 ng; Promega) using Lipofectamine $^{\text {TM }} 2000$ (Invitrogen; Thermo Fisher Scientific, Inc., Waltham, MA, USA) according to the manufacturer's protocol. Overexpression of GATA4 was achieved by transfecting the $\mathrm{T}$ vector (200 ng; Promega) containing the coding sequence of GATA4. At $48 \mathrm{~h}$ post-transfection, the luciferase activity was detected using the GloMax $®$-Multi Detection System (Promega) normalized against Renilla luciferase gene activity.

Immunohistochemistry and immunocytochemistry. Heart tissue from rats in the Control and TAAC groups were embedded in paraffin and cut into $4 \mu \mathrm{m}$ slices. A total of $1 \times 10^{5} \mathrm{CMs}$ were seeded onto the slide in 6 -well plates, and cultured and treated with Ang II as described above. The cells were fixed with $4 \%$ paraformaldehyde for $15 \mathrm{~min}$, and transparentized with $0.5 \%$ Triton X-100 for $20 \mathrm{~min}$. All the tissue and cell slides were incubated in $3 \% \mathrm{H}_{2} \mathrm{O}_{2}$ for $15 \mathrm{~min}$ at room temperature. The cells were blocked by incubation in goat serum (Sigma-Aldrich China, Inc.) for $30 \mathrm{~min}$, and subsequently the cells were incubated with a mouse monoclonal anti- $\alpha$-actinin antibody (1:1,000; Abcam; cat. no. ab108198) at $4^{\circ} \mathrm{C}$ overnight, and then with a goat anti-rabbit horseradish peroxidase (HRP)-conjugated secondary antibody $(1: 2,000$; Abcam; cat. no. ab7090) for $30 \mathrm{~min}$. Positive signals were developed using diaminobenzidine (Solarbio, Beijing, China) staining, after which the cells were counterstained with hematoxylin. The slides were observed under a microscope (MM200; Nikon, Tokyo, Japan), and the CM area was calculated using ImageJ version 1.49 (National Institutes of Health, Bethesda, MD, USA).

Leucine incorporation assay. Leucine incorporation assay was performed to reflect the protein synthesis in CMs. Following Ang II treatment, ${ }^{3} \mathrm{H}$-labeled leucine $(1 \mu \mathrm{Ci} / \mathrm{ml})$ was added to the cells for incubation at $37^{\circ} \mathrm{C}$ for $12 \mathrm{~h}$. Subsequently, the cells were washed three times with cold phosphate-buffered saline, and lysed in lysis buffer. The $\left[{ }^{3} \mathrm{H}\right]$ leucine signal was detected by liquid scintillation counting using Tri-Carb (PerkinElmer, Inc., Waltham, MA, USA). 
A

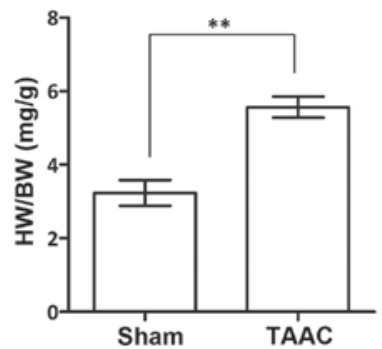

C

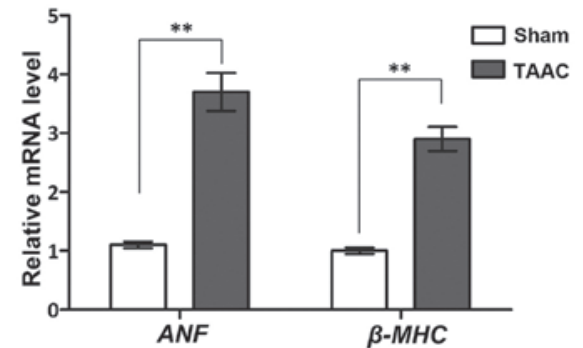

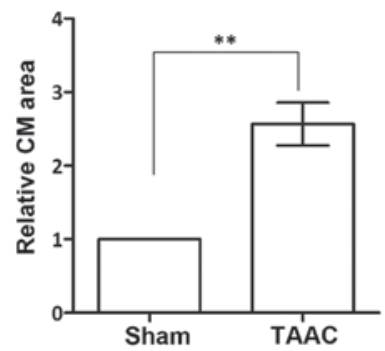

D

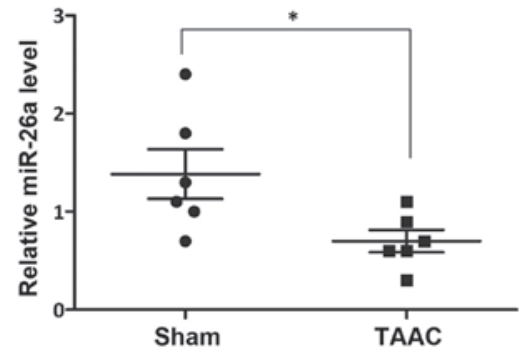

Figure 1. miR-26a inhibits cardiac hypertrophy in a TAAC-induced rat model. (A) The HW proportion, indicated by HW/BW, is larger in the TAAC group. (B) The relative CM area is larger in the TAAC group. (C) mRNA expression of $A N F$ and $\beta-M H C$ is upregulated in the TAAC group. (D) The miR-26a level is inhibited in the TAAC group. ${ }^{*} \mathrm{P}<0.05 ;{ }^{* *} \mathrm{P}<0.01$ vs. Sham group. $\mathrm{HW}$, heart weight. BW, body weight; miR-26a, microRNA-26a; CM, cardiomyocyte; ANF, atrial natriuretic factor; $\beta$-MHC, $\beta$-myosin heavy chain; TAAC, the cardiac hypertrophy rat model induced by TAAC surgery; Sham, the rats underwent the identical surgery procedures, except for TAAC; TAAC, transverse abdominal aortic constriction.

Reverse transcription-quantitative PCR (RT-qPCR). Total RNA was extracted from CMs and heart tissues using TRIzol (Invitrogen; Thermo Fisher Scientific, Inc.). First-strand complementary DNA (cDNA) was synthesized using a PrimeScript $^{\mathrm{TM}}$ 1st Strand cDNA Synthesis kit (Takara Bio, Dalian, China). For miRNA extraction and reverse transcription, RNAiso for Small RNA and a One Step PrimeScript miRNA cDNA Synthesis kit (both from Takara Bio) were used. RT-qPCR was performed using a LightCycler ${ }^{\circledR} 480$ system (Roche, Basel, Switzerland) with SYBR Green I master (Roche). Each reaction system consisted of $20 \mathrm{ng}$ cDNA, and the specific primers used were as follows: $\beta-M H C$ [forward (F), 5'-CCTCGCAATATCAAGGGAAA-3' and reverse (R), 5'-TACAGGTGCATCAGCTCCAG-3'], ANF (F, 5'-GCCGGT AGA AGATGAGGTCA-3' and R, 5'-GGGCTCCAATCC TGTCAATC-3'), GATA4 (F, 5'-GGGCGAGCCTGTTTG CAATG-3' and R, 5'-TGCTTGGAGCTGGCCTGTGA-3') or miR-26 (F, 5'-ATGGCTTCAAGTAATCC-3' and R, 5'-GTG CAGGGTCCGAGGT-3'). The thermocycling conditions were as follows: $95^{\circ} \mathrm{C}$ for $10 \mathrm{~min} ; 40$ cycles of $95^{\circ} \mathrm{C}$ for $20 \mathrm{sec}, 64^{\circ} \mathrm{C}$ for $30 \mathrm{sec}$ and $72^{\circ} \mathrm{C}$ for $30 \mathrm{sec}$; and a melting curve of $95^{\circ} \mathrm{C}$ for $15 \mathrm{sec}, 60^{\circ} \mathrm{C}$ for $1 \mathrm{~min}$ and $95^{\circ} \mathrm{C}$ for $15 \mathrm{sec}$. Data were analyzed using the $2^{-\Delta \Delta \mathrm{Cq}}$ method. Glyceraldehyde-3-phosphate dehydrogenase (GAPDH; F, 5'-CGCATTGCCAGACATATC AGC-3' and R, 5'-AGGTGAAGCAGGCTCAATCAA-3') and U6 (F, 5'-GCTTCGGCAGCACATATACTAAAAT-3' and R, 5'-CGCTTCACGAATTTGCGTGTCAT-3') were used as the internal reference compounds.

Western blot analysis. CMs and heart tissues were lysed in cold radioimmunoprecipitation assay (RIPA) buffer (Beyotime Institute of Biotechnology, Shanghai, China), and the extracted protein samples were quantified using the Bio-Rad protein assay kit (Bio-Rad, Hercules, CA, USA). Identical quantities of protein samples were separated using $10 \%$ sodium dodecyl sulfate-polyacrylamide gel electrophoresis and transferred on to a polyvinylidene fluoride membrane (Roche). The membrane was blocked with 5\% skimmed milk at room temperature for $2 \mathrm{~h}$, followed by an incubation with the specific primary antibody against GATA4 (1:1,000; Abcam; cat. no. ab86371) at $4^{\circ} \mathrm{C}$ overnight. GAPDH was used as the internal reference. Following washing three times for $5 \mathrm{~min}$ each time in Tris-buffered saline with Tween (TBST), the membrane was incubated with the HRP-conjugated secondary antibody at room temperature for $2 \mathrm{~h}$, and washed again in TBST. Subsequently, the positive bands were developed using the enhanced chemiluminescence (ECL) Plus Western Blotting substrate (Thermo Fisher Scientific, Inc.) and analyzed using Image J 1.49 software.

Statistical analysis. All the experiments were performed at least in triplicate, and results are expressed as the mean \pm standard deviation. Data were analyzed using one-way analysis of variance, followed by an unpaired t-test with SPSS 19.0 software (IBM SPSS, Armonk, NY, USA). P $<0.05$ was taken to indicate a statistically significant difference.

\section{Results}

miR-26a is downregulated in the cardiac hypertrophy rat model. Prior to analyzing the expression level of miR-26a in the cardiac hypertrophy rat model, the wet weight of the heart (in $\mathrm{mg}$ ) compared with the BW (g) at 4 weeks post-TAAC surgery was first determined. The results revealed a significantly larger proportion of heart tissue in the TAAC group compared with the Sham group $(\mathrm{P}<0.01$; Fig. 1A). The relative 
A

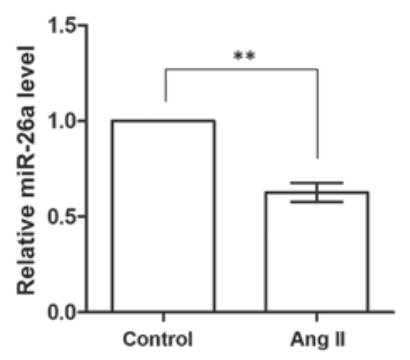

C

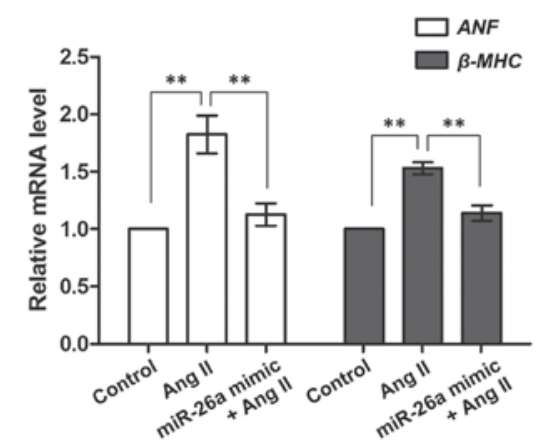

B

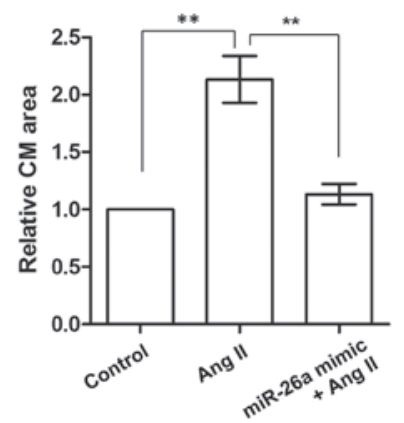

D

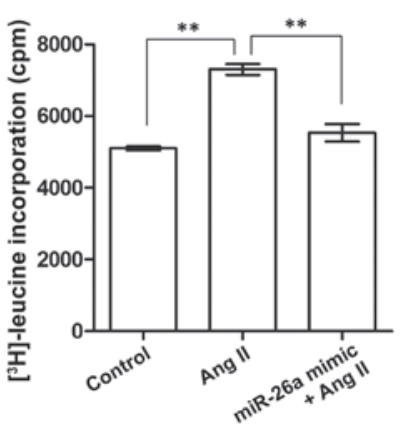

Figure 2. miR-26a inhibits cardiac hypertrophy in Ang II-induced CMs. (A) miR-26a expression is inhibited in Ang II-treated CMs. (B) The CM area is larger in Ang II-treated cells, but decreases with miR-26a overexpression. (C) Expression of $A N F$ and $\beta$-MHC mRNA is upregulated in Ang II-treated CMs, and downregulated by miR-26a overexpression. (D) Protein synthesis, indicated by $\left[{ }^{3} \mathrm{H}\right]$ leucine incorporation, is accelerated in Ang II-treated cells and inhibited by miR-26a overexpression. ${ }^{* *} \mathrm{P}<0.01$ vs. the Ang II group. Control group, CMs without Ang II treatment; Ang II group, CMs treated with Ang II to induce cardiac hypertrophy. miR-26a mimic group, CMs overexpressing miR-26a; CM, cardiomyocyte; Ang II, angiotensin II; miR-26a, microRNA-26a; ANF, atrial natriuretic factor. $\beta$-MHC, $\beta$-myosin heavy chain; $\mathrm{cpm}$, counts per min.

A

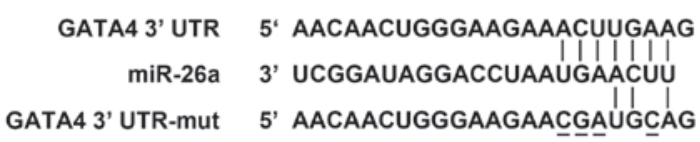

B

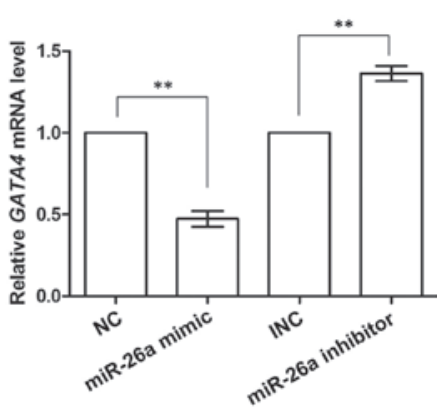

D

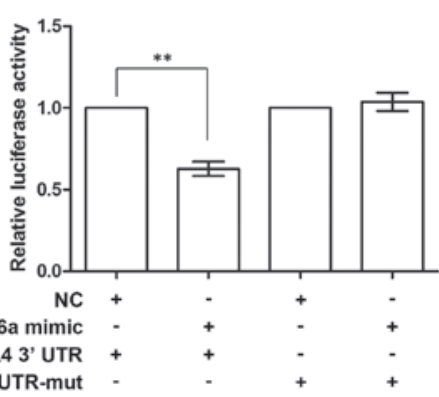

C
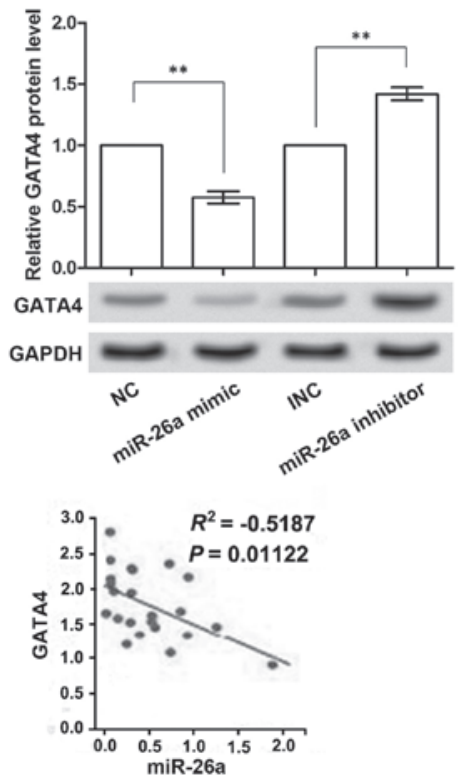

Figure 3. miR-26a directly suppresses GATA4 expression. (A) GATA4 is predicted to be the target gene of miR-26a by TargetScan. miR-26a can bind to positions 131-137 in the GATA4 3'-UTR. The mutant GATA4 3'-UTR (GATA3 3'-UTR-mut) was designed by mutation of four sites (which are shown underlined). (B) GATA4 mRNA expression is inhibited by miR-26a overexpression. (C) GATA4 protein expression is inhibited by miR-26a overexpression. GAPDH was the internal reference compound. (D) miR-26a targets the binding sites in GATA4 3'-UTR. No significant difference was found when mutating the GATA4 3'-UTR. (E) Correlation analysis between GATA4 and miR-26a expression. ${ }^{* *} \mathrm{P}<0.01$ compared with the Control group. GATA4, GATA-binding protein 4; NC, mimic negative control. INC, inhibitor negative control; miR-26a, microRNA-26a; UTR, untranslated region; GAPDH, glyceraldehyde-3-phosphate dehydrogenase. 
A

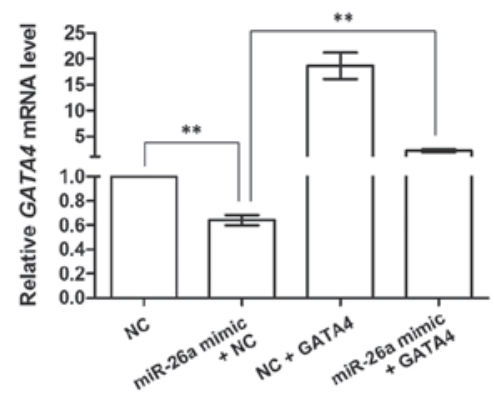

B

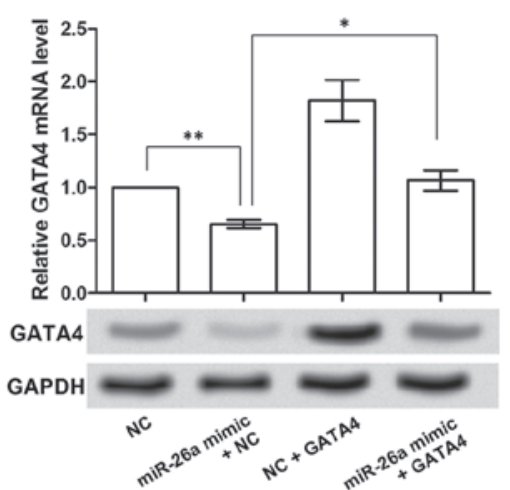

C

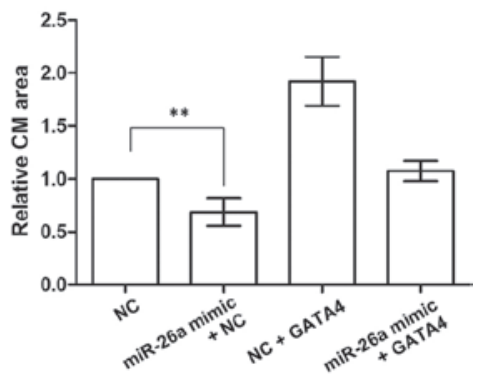

D

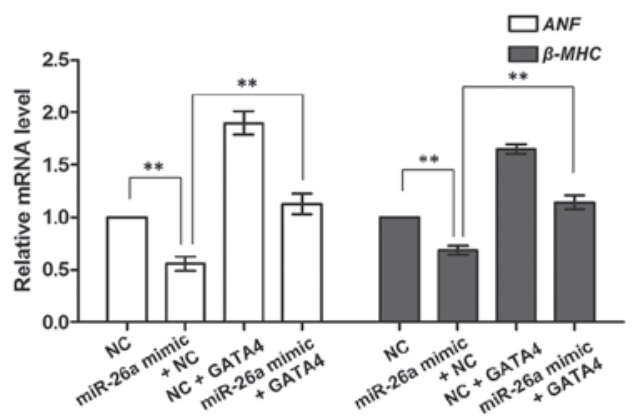

E

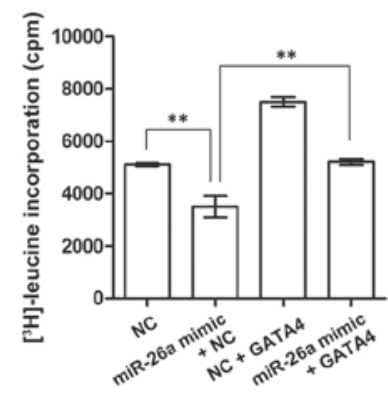

Figure 4. GATA4 compensates for the inhibitory functions of miR-26a in cardiac hypertrophy. (A) GATA4 mRNA expression is reversed by GATA4 overexpression when miR-26a is overexpressed. (B) GATA4 protein expression is reversed by GATA4 overexpression when miR-26a is overexpressed. GAPDH was used the internal reference. (C) The CM area is enlarged by GATA4 overexpression with miR-26a overexpression. (D) $A N F$ and $\beta$ - $M H C$ mRNA expression levels are upregulated by GATA4. (E) Protein synthesis, as determined by $\left[{ }^{3} \mathrm{H}\right]$ leucine incorporation, was promoted by GATA4. *P $<0.05$; ${ }^{* *} \mathrm{P}<0.01$ compared with the respective groups. CM, cardiomyocyte; ANF, atrial natriuretic factor; $\beta$-MHC, $\beta$-myosin heavy chain; GAPDH, glyceraldehyde-3-phosphate dehydrogenase; $\mathrm{NC}$, mimic negative control; cpm, count per min.

CM area, one of the characteristics of cardiac hypertrophy, was also examined. By observing the tissue sections, the $\mathrm{CMs}$ in the TAAC group were revealed to occupy larger areas $(\mathrm{P}<0.01$; Fig. 1B). These results suggested that the TAAC surgery had successfully induced cardiac hypertrophy in the rat model. Subsequently, two cardiac hypertrophy-associated genes, ANF and $\beta-M H C$, were analyzed in the Sham and TAAC groups to further verify the cardiac hypertrophy rat model (Fig. 1C). The mRNA expression levels of the two genes were significantly promoted in the TAAC group compared with the Sham group $(\mathrm{P}<0.01)$, which demonstrated that the rats in the TAAC group were afflicted with cardiac hypertrophy. Following this verification, the expression of miR-26a expression was detected, which was shown to be significantly downregulated in the TAAC group $(\mathrm{P}<0.05$, Fig. 1D). This result indicated that miR-26a was abnormally expressed in the heart tissue of the cardiac hypertrophy rat model, suggesting that miR-26a may serve as an important regulator in cardiac hypertrophy.

miR-26a inhibits cardiac hypertrophy in cultured CMs. The cultured CMs from neonatal rats were treated with Ang II to induce cardiac hypertrophy, after which it was determined whether the induction had been effective by detecting the expression of miR-26a, which was found in the above-mentioned results to be downregulated in the heart of the cardiac hypertrophy rat model. These results demonstrated that miR-26a was also inhibited in the Ang II-treated CMs compared with the Control group $(\mathrm{P}<0.01$, Fig. 2A), indicating that cardiac hypertrophy had been successfully induced in the cultured CMs on Ang II treatment. Subsequently, miR-26a was overexpressed to examine its influence on cardiac hypertrophic CMs. The CM area was significantly enlarged, and the expression levels of $A N F$ and $\beta-M H C$ mRNA were significantly upregulated, on Ang II treatment $(\mathrm{P}<0.01$; Fig. $2 \mathrm{~B}$ and $\mathrm{C})$, although these were restored to normal levels on miR-26a overexpression $(\mathrm{P}<0.01)$. $\left[{ }^{3} \mathrm{H}\right]$ leucine incorporation was also measured to assess the protein synthesis rate in CMs, since an accelerated protein synthesis is another characteristic of cardiac hypertrophy. These results demonstrated that protein synthesis was enhanced in Ang II-treated CMs, although this enhancement was diminished by miR-26a overexpression. Taken together, these results indicated that CMs induced by Ang II exhibited cardiac hypertrophic properties, such as a larger CM area, accelerated protein synthesis and promotion of the expression of cardiac hypertrophy-associated genes. However, overexpression of miR-26a was able to significantly inhibit these effects, implying that it exerts a role in inhibiting cardiac hypertrophy in cultured CMs.

GATA4 is the direct target gene of miR-26a. GATA4 was predicted to be the target gene of miR-26a by TargetScan (Fig. 3A). Therefore, the impact of miR-26a on GATA4 expression using the miR-26a mimic and inhibitor was analyzed. The RT-qPCR results demonstrated that the expression of GATA4 mRNA was inhibited when overexpressing miR-26a $(\mathrm{P}<0.01$; Fig. 3B), and was promoted when miR-26a was inhibited $(\mathrm{P}<0.01)$, compared with the corresponding control group. Similarly, the protein expression of GATA4 was also down- 
regulated or upregulated with the promotion or inhibition of miR-26a expression, respectively $(\mathrm{P}<0.01$; Fig. $3 \mathrm{C})$. These data confirmed the prediction that GATA4 was the target gene of miR-26a.

To investigate whether GATA4 was directly inhibited by miR-26a, four sites in the predicted binding sites (positions 131-137 of 3'-UTR) in GATA4 were mutated (Fig. 3A). miR-26a mimic was co-transfected with the wild-type or mutant GATA4 3'-UTR, and the luciferase activities were measured (Fig. 3D). These results showed that, when overexpressing wild-type GATA4 3'-UTR and miR-26a, the activity of the luciferase reporter gene linked with the wild-type GATA4 3'-UTR significantly decreased $(\mathrm{P}<0.01)$. However, the activity of the mutant GATA4 3'-UTR was almost unchanged on miR-26a overexpression. Multiple data groups were used for analyzing the correlation between GATA4 and miR-26a expression (Fig. 3E), and these results revealed that there was a significant negative correlation. Taken together, these data indicated that miR-26a was able to directly bind to and suppress GATA4.

GATA4 compensates for the suppressive roles of miR-26a in cardiac hypertrophy. In the above-mentioned experiments, GATA4 was demonstrated to be the direct target gene of miR-26a. It was also hypothesized that GATA4 may be involved in regulating cardiac hypertrophy. The overexpression of GATA4 in CMs was therefore investigated on the basis of miR-26a overexpression to investigate its functions in cardiac hypertrophy. Prior to performing this experiment, the overexpression of GATA4 was determined to be valid (Fig. 4A and $\mathrm{B}$ ). These results indicated that miR-26a overexpression significantly inhibited the mRNA and protein expression of GATA4 $(\mathrm{P}<0.01)$, and subsequent overexpression of GATA4 was able to increase the inhibited GATA4 mRNA and protein levels caused by miR-26a $(\mathrm{P}<0.01$ or $\mathrm{P}<0.05)$. Therefore, the overexpression of GATA4 was shown to be effective for further analyses.

Subsequently, the role of GATA4 in cardiac hypertrophy was investigated by analyzing the CM area, hypertrophy-associated gene expression and protein synthesis. The CM area was decreased by miR-26a overexpression, and increased by GATA4 overexpression alone $(\mathrm{P}<0.01)$. It also appeared to be modestly enlarged by GATA4 overexpression when miR-26a was also overexpressed, although not significantly so $(\mathrm{P}>0.05$, Fig. 4C). Similarly, the expression of the hypertrophy-associated genes, $A N F$ and $\beta-M H C$, and the protein synthesis rate, as determined by $\left[{ }^{3} \mathrm{H}\right]$ leucine incorporation, were all inhibited by miR-26a, but promoted by GATA4 overexpression $(\mathrm{P}<0.01$; Fig. 4D and E), even with the overexpression of miR-26a. Taken together, as the target gene inhibited by miR-26a, GATA4 overexpression could compensate for the suppressive functions of miR-26a in cardiac hypertrophy, suggesting that GATA4 may be a facilitator of cardiac hypertrophy.

\section{Discussion}

In the present study, the anti-cardiac hypertrophic roles of miR-26a were investigated. miR-26a is expressed at lower levels in the heart of cardiac hypertrophy rat model, although its overexpression, however, causes a significant protection against cardiac hypertrophy in CMs via inhibition of GATA4.
GATA4 was demonstrated to be the direct target of miR-26a, exerting a pro-hypertrophic role, since its overexpression leads to aggravated cardiac hypertrophy in CMs.

miRNAs exert significant roles in the progression of cardiac hypertrophy. A variety of miRNAs show differential and temporal expression patterns during cardiac hypertrophy, such as miR-1, miR-149 and miR-10a, amongst others, of which downregulation of miR-26a may be detected at $\sim 14$ days post-TAAC (17). Thus, in the present study, experiments were performed at 4 weeks post-TAAC in the rat model, and miR-26a was revealed to be significantly inhibited, both in the TAAC-induced rat model and in the cultured rat CMs following $48 \mathrm{~h}$ of Ang II treatment. As elsewhere reported, miR-26a/b may perform distinct functions in different diseases, not only oncogenic roles, but also tumor-suppressive roles (18). However, miR-26b has been shown to be able to inhibit GATA4 and suppress pressure-induced cardiac hypertrophy (19). Additionally, miR-26a/b has been revealed to be an anti-hypertrophy factor via the suppression of glycogen synthase kinase $3 \beta$ (20). In accordance with these findings, the hypertrophy-suppressive roles of miR-26a identified in the present study of the TAAC-induced rat model and Ang II-treated cells disclosed a further signaling pathway involving miR-26a via GATA4, revealing a novel regulatory mechanism for miR-26a.

In these experiments, $\mathrm{HW} / \mathrm{BW}$, the $\mathrm{CM}$ area, and expression of hypertrophy-associated genes were used as indicators of cardiac hypertrophy; protein synthesis, as measured by leucine incorporation assay, was also included when monitoring changes in CMs. Based on the aforementioned knowledge that cardiac hypertrophy is usually manifested as larger volumes and accelerated protein synthesis in CMs, the results of the present study revealed that the TAAC-induced rat model exhibited larger hearts, with a higher HW/BW ratio, and larger $\mathrm{CMs}$, as indicated by their larger relative area. The cultured CMs isolated from neonatal rats also possessed a larger area, as well as accelerated protein synthesis, when treated with Ang II, a factor that can increase protein synthesis and cause hypertrophy of vascular smooth muscle cells $(21,22)$. ANF and $\beta$-MHC were used as indices of cardiac hypertrophy in the present study for correlation of their expression with cardiac hypertrophy, and upregulated expression levels were identified compared with normal heart tissues (23). Switching from $\alpha$-MHC to $\beta$-MHC causes a higher expression level of $\beta$-MHC during hypertrophy (24). The levels of ANF and $\beta$-MHC are mediated by various regulators and signaling pathways. Previous studies have demonstrated the association between GATA4 and its downstream factors, including ANF and $\beta$-MHC, which are activated by GATA 4 by binding of this protein at sites in their promoter region $(25,26)$. Similarly, in the present study, overexpression of GATA4 could upregulate $\mathrm{ANF}$ and $\beta$-MHC in the TAAC-induced rat model and Ang II-induced rat CMs, which indicated that ANF and $\beta-\mathrm{MHC}$ were possible downstream targets of GATA4, constituting a vital signaling pathway in cardiac hypertrophy regulation.

As an important transcription factor closely associated with heart development, GATA4 is the integrator of several signaling pathways in regulating cardiac hypertrophy. In addition to the above-mentioned ANF and $\beta$-MHC, GATA4 is also able to regulate the expression of B-type natriuretic 
peptide in CMs, possibly via the GATA4/nuclear factor of activated T-cells-3 signaling pathway (27). Several factors regulating GATA4 in cardiac hypertrophy have been reported. Transcription factor CHF-1/Hey2 binds GATA4 to inhibit the regulation of GATA4 on the ANF promoter (28), whereas estrogen-related receptor $\gamma$ activates GATA4 to exacerbate cardiac hypertrophy (29). Other upstream mediators of GATA4 include the MEK1/ERK1/2 signaling pathway, where ERK2 directly phosphorylates GATA4 (30), and miRNAs such as miR-208a, which controls the expression of GATA4 during cardiac hypertrophy (10). In the present study, miR-26a was shown to inhibit GATA4 by targeting the binding sites in the 3'-UTR, since the mutated GATA4 3'-UTR did not achieve successful regulation by miR-26a. Taken together, GATA4 was able to regulate various downstream factors, which were likely to be controlled by proteins and miRNAs upstream, forming a part of the regulatory network during cardiac hypertrophy.

In summary, the present study has identified a protective role of miR-26a via suppression of GATA4, and, in turn, further downstream factors in cardiac hypertrophy. These results are potentially useful in terms of the possible use of miR-26a, and therapeutic targets such as GATA4, in cardiac hypertrophy treatment.

\section{References}

1. Senthil V, Chen SN, Tsybouleva N, Halder T, Nagueh SF, Willerson JT, Roberts R and Marian AJ: Prevention of cardiac hypertrophy by atorvastatin in a transgenic rabbit model of human hypertrophic cardiomyopathy. Circ Res 97: 285-292, 2005.

2. Okere IC, Young ME, McElfresh TA, Chess DJ, Sharov VG, Sabbah HN, Hoit BD, Ernsberger P, Chandler MP and Stanley WC: Low carbohydrate/high-fat diet attenuates cardiac hypertrophy, remodeling and altered gene expression in hypertension. Hypertension 48: 1116-1123, 2006.

3. Doyle B, Sorajja P,Hynes B,Kumar AH,AraozPA, Stalboerger PG, Miller D, Reed C, Schmeckpeper J, Wang S, et al: Progenitor cell therapy in a porcine acute myocardial infarction model induces cardiac hypertrophy, mediated by paracrine secretion of cardiotrophic factors including TGFbeta1. Stem Cells Dev 17: 941-951, 2008.

4. Weeks KL and McMullen JR: The athlete's heart vs. the failing heart: Can signaling explain the two distinct outcomes? Physiology (Bethesda) 26: 97-105, 2011.

5. Kodama H, Fukuda K, Pan J, Sano M, Takahashi T, Kato T, Makino S, Manabe T, Murata M and Ogawa S: Significance of ERK cascade compared with JAK/STAT and PI3-K pathway in gp130-mediated cardiac hypertrophy. Am J Physiol Heart Circ Physiol 279: H1635-H1644, 2000.

6. Lezoualc'h F, Metrich M, Hmitou I, Duquesnes N and Morel E: Small GTP-binding proteins and their regulators in cardiac hypertrophy. J Mol Cell Cardiol 44: 623-632, 2008.

7. Pan J, Singh US, Takahashi T, Oka Y, Palm-Leis A, Herbelin BS and Baker KM: PKC mediates cyclic stretch-induced cardiac hypertrophy through Rho family GTPases and mitogen-activated protein kinases in cardiomyocytes. J Cell Physiol 202: 536-553, 2005.

8. Yue H, Li W, Desnoyer R and Karnik SS: Role of nuclear unphosphorylated STAT3 in angiotensin II type 1 receptor-induced cardiac hypertrophy. Cardiovasc Res 85: 90-99, 2010.

9. Gladka MM, da Costa Martins PA and De Windt LJ: Small changes can make a big difference - microRNA regulation of cardiac hypertrophy. J Mol Cell Cardiol 52: 74-82, 2012.

10. Callis TE, Pandya K, Seok HY, Tang RH, Tatsuguchi M, HuangZP, Chen JF, Deng Z, Gunn B, Shumate J, et al: MicroRNA-208a is a regulator of cardiac hypertrophy and conduction in mice. J Clin Invest 119: 2772-2786, 2009.
11. Wang K, Lin ZQ, Long B, Li JH, Zhou J and Li PF: Cardiac hypertrophy is positively regulated by MicroRNA miR-23a. J Biol Chem 287: 589-599, 2012.

12. Huang ZP, Chen J, Seok HY, Zhang Z, Kataoka M, Hu X and Wang DZ: MicroRNA-22 regulates cardiac hypertrophy and remodeling in response to stress. Circ Res 112: 1234-1243, 2013.

13. Care A, Catalucci D, Felicetti F, Bonci D, Addario A, Gallo P, Bang ML, Segnalini P, Gu Y, Dalton ND, et al: MicroRNA-133 controls cardiac hypertrophy. Nat Med 13: 613-618, 2007.

14. Li Q, Song XW, Zou J, Wang GK, Kremneva E, Li XQ, Zhu N, Sun T, Lappalainen P, Yuan WJ, et al: Attenuation of microRNA-1 derepresses the cytoskeleton regulatory protein twinfilin-1 to provoke cardiac hypertrophy. J Cell Sci 123: 2444-2452, 2010.

15. Wei L, Yuan M, Zhou R, Bai Q, Zhang W, Zhang M, Huang Y and Shi L: MicroRNA-101 inhibits rat cardiac hypertrophy by targeting Rabla. J Cardiovasc Pharmacol 65: 357-363, 2015.

16. Cheng Y, Ji R, Yue J, Yang J, Liu X, Chen H, Dean DB and Zhang C: MicroRNAs are aberrantly expressed in hypertrophic heart: Do they play a role in cardiac hypertrophy? Am J Pathol 170: 1831-1840, 2007.

17. Sayed D, Hong C, Chen IY, Lypowy J and Abdellatif M: MicroRNAs play an essential role in the development of cardiac hypertrophy. Circ Res 100: 416-424, 2007.

18. Gao J and Liu QG: The role of miR-26 in tumors and normal tissues (Review). Oncol Lett 2: 1019-1023, 2011.

19. Han M, Yang Z, Sayed D, He M, Gao S, Lin L, Yoon S and Abdellatif M: GATA4 expression is primarily regulated via a miR-26b-dependent post-transcriptional mechanism during cardiac hypertrophy. Cardiovasc Res 93: 645-654, 2012.

20. Zhang ZH, Li J, Liu BR, Luo CF, Dong Q, Zhao LN, Zhong Y, Chen WY, Chen MS and Liu SM: MicroRNA-26 was decreased in rat cardiac hypertrophy model and may be a promising therapeutic target. J Cardiovasc Pharmacol 62: 312-319, 2013.

21. Parmentier JH,Pavicevic Z and Malik KU: Ang II stimulates phospholipase D through PKCzeta activation in VSMC: Implications in adhesion, spreading and hypertrophy. Am J Physiol Heart Circ Physiol 290: H46-H54, 2006.

22. Li R, Xiao J, Qing X, Xing J, Xia Y, Qi J, Liu X, Zhang S, Sheng X, Zhang X and Ji X: Sp1 mediates a therapeutic role of MiR-7a/b in angiotensin II-induced cardiac fibrosis via mechanism involving the TGF- $\beta$ and MAPKs pathways in cardiac fibroblasts. PLoS One 10: e0125513, 2015 .

23. Garcia R, Thibault $\mathrm{G}$ and Cantin M: Correlation between cardiac hypertrophy and plasma levels of atrial natriuretic factor in non-spontaneous models of hypertension in the rat. Biochem Biophys Res Commun 145: 532-541, 1987.

24. Haddad F, Qin AX, Bodell PW, Zhang LY, Guo H, Giger JM and Baldwin KM: Regulation of antisense RNA expression during cardiac MHC gene switching in response to pressure overload. Am J Physiol Heart Circ Physiol 290: H2351-H2361, 2006.

25. Hu X, Li T, Zhang C, Liu Y, Xu M, Wang W, Jia Z, Ma K, Zhang Y and Zhou C: GATA4 regulates ANF expression synergistically with Sp1 in a cardiac hypertrophy model. J Cell Mol Med 15: 1865-1877, 2011

26. Charron F, Paradis P, Bronchain O, Nemer G and Nemer M: Cooperative interaction between GATA-4 and GATA-6 regulates myocardial gene expression. Mol Cell Biol 19: 4355-4365, 1999.

27. Liu YL, Huang CC, Chang CC, Chou CY, Lin SY, Wang IK, Hsieh DJ, Jong GP, Huang CY and Wang CM: Hyperphosphate-induced myocardial hypertrophy through the GATA-4/NFAT-3 signaling pathway is attenuated by ERK inhibitor treatment. Cardiorenal Med 5: 79-88, 2015.

28. Xiang F, Sakata Y, Cui L, Youngblood JM, Nakagami H, Liao JK, Liao R and Chin MT: Transcription factor CHF1/Hey2 suppresses cardiac hypertrophy through an inhibitory interaction with GATA4. Am J Physiol Heart Circ Physiol 290: H1997-H2006, 2006.

29. Kwon DH, Eom GH, Kee HJ, Nam YS, Cho YK, Kim DK, Koo JY, Kim HS, Nam KI, Kim KK, et al: Estrogen-related receptor gamma induces cardiac hypertrophy by activating GATA4. J Mol Cell Cardiol 65: 88-97, 2013.

30. Liang Q, Wiese RJ, Bueno OF, Dai YS, Markham BE and Molkentin JD: The transcription factor GATA4 is activated by extracellular signal-regulated kinase 1- and 2-mediated phosphorylation of serine 105 in cardiomyocytes. Mol Cell Biol 21: 7460-7469, 2001. 